\title{
Fagfellevurderere 2013
}

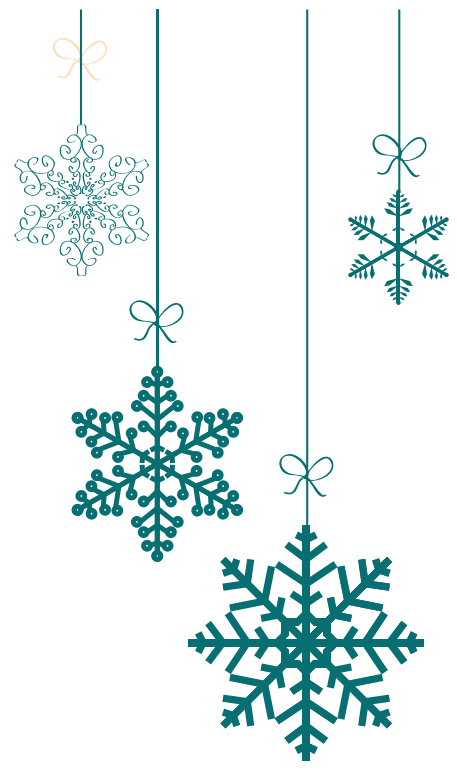

Akselsen, Per

Aksnes, Dag

Aksnes, Håvard

Alfsen, G. Cecilie

Alstadhaug, Karl B.

Angelsen, Anders

Aurlien, Dag

Bachmann, Ingeborg

Backe, Bjørn

Bakke, Søren

Balteskard, Lise

Bastholt, Lars

Bendiksen, Cecilie

Berentsen, Sigbjørn

Berg, Tore Julsrud

Bergh, Sverre

Bergsholm, Per

Berild, Dag

Berntsen, Erik Magnus

Berstad, Audun

Bindoff, Laurence

Birkeland, Kåre

Bjerkestrand, Stine

Bjørgul, Kristian

Bjørnelv, Sigrid

Bjørner, Trine

Blom, Hans

Bosse, Gerhard

Brandstorp, Helen

Bratland, Svein

Brekke, Mette

Bretthauer, Michael
Brodal, Per

Brodtkorb, Eylert

Brodwall, Kristoffer

Brox, Jens

Brudvik, Christina

Bruland, Øyvind

Brustad, Magritt

Bruun Wyller, Vegard

Bruun, Johan

Brækhus, Anne

Bugge, Jan

Bøhmer, Ellen

Calisch, Tor Einar

Castberg, Ingrid

Celius, Elisabeth

Cooper, John

Dahl, Vegard

Dale, Ola

Dalgaard, Jakob

De Lange, Charlotte

Due, Jan

Due-Tønnessen, Paulina

Ebbing, Cathrine

Eikeland, Randi

Ekeberg, Øivind

Ekerhovd, Erling

Elgen, Irene

Ellingsen, Christian

Elverland, Hans

Engelsen, Bernt

Eri, Lars

Eskild, Anne

Essén, Birgitta

Fetveit, Arne

Fevang, Jonas

Flaatten, Hans

Fors, Egil

Fosse, Gunilla Klensmeden

Fosse, Roar

Fosså, Alexander

Fosså, Kristian

Fretheim, Atle

Frich, Jan

Friis, Svein

Frøen, Frederik

Furberg, Anne-Sofie

Fure, Brynjar

Garåsen, Helge

Getz, Linn

Gisvold, Sven Erik

Gjennestad, Anne Lise

Gjersing, Linn
Gjesdal, Knut

Gjesdal, Sturla

Gjessing, Håkon

Godt, Kristine

Graff-Iversen, Sidsel

Gram, Inger Torhild

Gran, Jan

Grill, Valdemar

Grimsrud, Tom Kristian

Grøholt, Berit

Grøndahl, Pål

Grønn, Morten

Gudim, Hilde Beate

Gudmundsdottir, Helga

Gulati, Sasha

Gulbrandsen, Pål

Gulseth, Hanne

Gunnarsson, Ragnar

Haarr, Dagfinn

Haaverstad, Rune

Hagberg, Hans

Hagen, Bjørn

Hagen, Gaute

Hagen, Nils

Hager, Anders

Hagestad, Kristian

Hald, John

Hanoa, Rolf

Hansen, John-Bjarne

Hansson, Johan

Hartmann, Anders

Hasle, Gunnar

Haug, Jon

Haugaa, Kristina

Hauge, Truls

Haugen, Dagny Faksvåg

Haukeland, Jon

Haukås, Einar

Helgeland, Jon

Helland, Åslaug

Helle, Svein-Inge

Helseth, Eirik

Herfjord, Jo Kåre

Hilt, Bjørn

Hjelmesæth, Jøran

Hofoss, Dag

Hofstad, Bjørn

Holck, Per

Holmen, Jostein

Holmøy, Trygve

Holte, Harald

Horn, Morten

Houge, Gunnar

Hunskår, Steinar

Hunskår, Steinar

Høyersten, Jon
Iner, Stein Opjordsmoen Inchley, Christopher Indredavik, Bent Ingvaldsen, Baard Irgens, Lorentz Irtun, Øivind Iversen, Hilde Iversen, Ole-Erik Iversen, Per

Jahnsen, Jørgen Jensen, Svein Arthur Jenssen, Trond Geir Johansen, May-Lill Johansen, Truls Johnsen, Erik Johnsen, Harald Johnsen, Roar Juel, Niels Gunnar Jørgensen, Anders Palmstrøm

Kalager, Mette Kerty, Emilia

Kessler, Ute

Kielland, Knut

Kirkengen, Anna

Kiserud, Torvid Kjekshus, Lars Erik Knardahl, Stein Kran, Anne-Marte Kringlen, Einar Kristiansen, Ivar Sønbø Krogseth, Maria Krohg-Sørensen, Kirsten Krohn, Jørgen Kvalem, Helen E. Kvarstein, Gunnvald Kvernmo, Hebe Kvistad, Kjell Kvåle, Rune Kørner, Hartwig

Ladstein, Rita Lagerløv, Per Lang, Astri Maria Larsen, Øivind

Larsen, Alf

Larsen, Alf Larsen, Tor Ketil Lassen, Kristoffer Laugsand, Eivor Lie, Anne Kveim Lie, Elisabeth Lien, Lars Lillebø, Atle Lindahl, Anne Karin 
Line, Paal-Dag Lundervold, Astrid Lundgren, Steinar Lyngø, Inger-Johanne Løkeland, Mette

Madsen, Steinar Magelssen, Morten Magnussen, Jon Markestad, Trond J. Martinsen, Tom Mathiesen, Ellisiv Mehlum, Lars Meland, Eivind Melle, Ingrid Mellgren, Gunnar Midelfart, Anna Midtbu, Jo-Endre Moen, Mette Morken, Nils-Halvdan Moseng, Dagfinn Moseng, Ole Georg Mossige, Svein Mowe, Morten Mygland, Åse Mæland, John Gunnar Mørland, Jørg

Nakken, Karl Otto Nakling, Jakob Nakstad, Per Natås, Olav

Ness, Ewa Nessa, John Nielsen, Erik Nielsen, Rune Nilsen, Kristian Nordrehaug, Jan Erik Næss, Øyvind

Omdal, Roald Omvik, Per Opdal, Mimi Ose, Leiv

Paulsen, Vemund Pedersen, Roar

Perminow, Gøri

Pihlstrøm, Lasse

Pukstad, Brita

Qvigstad, Elisabeth Qvigstad, Gunnar

Raknes, Guttorm Randem, Britt Randsborg, Per-Henrik
Rao, Vidar

Ree, Anne Hansen

Reikvam, Åsmund

Reimers, Arne

Risøe, Cecilie

Rogde, Sidsel

Rosenqvist, Randi

Rosvold, Elin Olaug

Ruths, Sabine

Ruud, Ellen

Ruud, Torleif

Røkkum, Magne

Saltvedt, Ingvild

Salvesen, Rolf

Sandset, Per Morten

Sandvei, Per

Sandvik, Leiv

Schiøtz, Aina

Schjesvold, Fredrik Hellem

Simonsen, Gunnar Skov

Simonsen, Anne

Sivertsen, Einar Andreas

Skeie, Ivar

Skeie, Geir Olve

Skivenes, Marit

Skjeldal, Ola $\mathrm{H}$.

Skovlund, Eva

Skårderud, Finn

Solberg, Berge

Solberg, Steinar

Solevåg, Anne Lee

Sorteberg, Angelika

Spigset, Olav

Staff, Annetine

Steen, Knut

Steen, Petter Andreas

Steigen, Terje

Steinsvåg, Sverre

Storstein, Anette

Straand, Jørund

Strømme, Petter

Stubhaug, Bjarte

Sundsfjord, Arnfinn

Sælensminde, Kjartan

Sørbye, Sveinung

Søreide, Jon Arne

Søreide, Kjetil

Thomassen, Lars

Thoresen, Magne

Tjora, Aksel

Tjønnfjord, Geir

Torfoss, Dag

Torgersen, Terje

Tveit, Arnljot
Udal, Anne Helseth

Ulstein, Ingun

Ulvestad, Elling

Urheim, Stig

Vaaler, Arne

Vogt, Christina

von Lueder, Thomas

Våge, Villy

Waal, Helge

Wesenberg, Finn

Wester, Knut Gustav

Westin, Andreas

Wie, Ona

Wiseth, Rune

Woldseth, Berit

Wyller, Torgeir

\section{Zeiner, Pål}

Øiesvold, Terje

$\emptyset$ vrebø, Kjell

Aabakken, Lars

Aaberge, Ingeborg

Aaberge, Lars

Aamodt, Arild

Aanderud, Sylvi

Aarø, Leif Edvard

Aase, Steinar

Åsberg, Arne

Aasebø, Ulf

Aasheim, Erlend

Aasly, Jan

Aavitsland, Preben 